\title{
Constructing the Spectrum of Packings and Coverings for the Complete Graph with Stars with up to Five Edges
}

\author{
Danny Dyer*, Sadegheh Haghshenas ${ }^{\dagger}$, and Nabil Shalaby ${ }^{\ddagger}$ \\ Department of Mathematics and Statistics, Memorial University of Newfoundland, St. John's, \\ Newfoundland, Canada, A1C 5Sr
}

\begin{abstract}
The packing and covering problems have been considered for several classes of graphs. For instance, Bryant et. al. have investigated the packing problem for paths and cycles, and the packing and covering problems for 3-cubes. The packing and covering problems were settled for stars with up to six edges by Roditty. In this paper, for every possible leave graph (excess graph), we find a corresponding maximum packing (minimum covering) of the complete graph with stars with up to five edges.
\end{abstract}

Keywords: Packing; Covering; Leave graph; Excess graph

\section{Introduction}

The graphs in this paper are assumed to have no loops or multiple edges unless otherwise stated. For any graph $G$, a $G$-decomposition of a graph $H$ is a partition of the edge set of $H$ with graphs all isomorphic to $G$. The spectrum problem for a graph $G$ is to determine the set $D$ of all positive integers $n$ such that the complete graph $K_{n}$ has a $G$-decomposition if and only if $n \in D$. In 1972, Hell and Rosa introduced graph decompositions for the first time in order to attack the spectrum problem for $P_{3}$, a path on three vertices [7].

The spectrum problem for trees on nine or less vertices was solved by Huang and Rosa in 1978 [9]. This problem has been completely solved for the stars, an infinite subclass of trees. Yamamoto solved the spectrum problem for stars with $k$ edges in 1975 [18]. In 1978, Tarsi [14] solved the problem using a different method.

The packing problem (covering problem) for a graph $G$ is to determine the number of elements in a maximum $G$-packing (minimum $G$-covering) of $K_{n}$ and this number is called the $G$-packing number ( $G$-covering number). The packing and covering problems have been considered for many classes of graphs. These problems were solved for all trees of order seven or less by Roditty [10], [11], [12], and [13]. In particular, he solved the problems for stars with up to six edges. In 1999, Adams, Bryant, and El-Zanati [2] solved the packing and

\footnotetext{
*dyer@mun.ca

†ssh631@mun.ca

${ }^{\ddagger}$ nshalaby@mun.ca
} 
covering problems for 3-cubes. In 2008, Bryant and Horsley [4] found sufficient conditions for the existence of a packing of the complete graph with cycles of specific lengths. Bryant [3] also proved Tarsi's conjecture [15] on necessary and sufficient conditions for the existence of a packing of the complete multigraph with paths of specific lengths in 2010. For further details about decompositions, packings, and coverings, refer to [5].

In 2014, Hoffman solved the packing and covering problems for any $k$-star [8]. In fact, he proved that for $n \geq 2 k$, the number of $k$-stars in a maximum $S_{k}$-packing of $K_{n}$ is $\left\lfloor\frac{n(n-1)}{2 k}\right\rfloor$, and a star is always achievable as the leave graph.

As mentioned above, Roditty solved the packing and covering problems for stars with up to six edges; however, he did not achieve all the possible leaves and excesses, which we refer to as the spectrum problem for packing and covering.

In 2013, Dyer, Haghshenas, and Shalaby solved the spectrum problem for packing and covering of the complete graph with stars with four edges [6]. In this paper, we solve the problem for stars with five edges and introduce new methods to shorten the proofs of the main theorems. We state these methods in the form of lemmas in the next section. We also believe these lemmas will help us to achieve results for the general case, stars with $k$ edges, in the future.

\section{Preliminaries}

For basic graph definitions, we follow [16]. Certainly, for a $G$-decomposition of $K_{n}$ to exist, the number of vertices of $K_{n}$ must be more than the number of vertices of $G$, the number of edges of $G$ need to divide the number of edges of $K_{n}$, and the degree of each vertex in $K_{n}$ has to be a multiple of $d$, the greatest common divisor of all vertex degrees in $G$. Consequently, the following are the obvious necessary conditions for the existence of a $G$-decomposition of $K_{n}$.

- $|V(G)| \leq n$ for $n>1$

- $n(n-1) \equiv 0(\bmod 2|E(G)|)$

- $n-1 \equiv 0(\bmod d)$ where $d$ is the greatest common divisor of the degrees of the vertices in $G$

These conditions were proved to be asymptotically sufficient by Wilson in 1975 [17]. However, in order to solve the spectrum problem thoroughly, it is still necessary to find the smallest number $n$ such that for all integers greater than or equal to $n$, these necessary conditions are sufficient. For results about those classes of graphs for which the spectrum problem has been solved refer to [1].

A connected graph on $k+1$ vertices with one vertex of degree $k$, called the center, and $k$ vertices of degree 1 , called the leaves, is a $k$-star and is denoted by $S_{k}$. We denote a $k$-star with the center $x$ and the leaves $y_{1}, y_{2}, \ldots, y_{k}$ by $\left(x ; y_{1}, y_{2}, \ldots, y_{k}\right)$. 
Theorem 1 ([18]). For $k \geq 1, K_{n}$ has an $S_{k}$-decomposition if and only if $n=1$, or $n \geq 2 k$ and $n(n-1) \equiv 0(\bmod 2 k)$.

In the case of the non-existence of a $G$-decomposition of a graph $H$, we are interested in getting as close as possible to a decomposition, from which arise the notions of packing and covering. A set of subgraphs of $H$ such that each subgraph is isomorphic to $G$ and every edge of $H$ is contained in at most one subgraph is a $G$-packing of $H$. The leave graph is the graph consisting of those edges of $H$ which are included in none of these subgraphs. A $G$-packing is a maximum $G$-packing if it has the smallest possible number of edges in the leave graph. A set of subgraphs of $H$ such that each subgraph is isomorphic to $G$ and every edge of $H$ is contained in at least one subgraph is a $G$-covering of $H$. For any $G$-covering, $C$, of $H$, the excess graph is the multigraph $\mathcal{C} \backslash H$ where $\mathcal{C}$ is the graph obtained from the union of all the subgraphs in the covering $C$. A $G$-covering is called a minimum $G$-covering if it has the smallest possible number of edges in the excess graph.

For any graph $G$, the number of elements in a maximum $G$-packing (minimum $G$-covering) of a graph $H$, is the $G$-packing number ( $G$-covering number) of $H$.

Theorem 2 ([10], [11], [12], and [13]). For integers $n$ and $k$, if $n \geq 2 k-1$ and $k \leq 6$, then the $S_{k}$-packing number of the complete graph $K_{n}$ is $\left\lfloor\frac{n(n-1)}{2 k}\right\rfloor$ and if $n \geq 2 k$ and $k \leq 6$, then the $S_{k}$-covering number of $K_{n}$ is $\left\lceil\frac{n(n-1)}{2 k}\right\rceil$.

We will use the following lemmas in the proof of the main theorems. These lemmas help us shorten the proofs in comparison with the proofs given in [6].

Lemma 3. If $m, n$, and $k$ are positive integers, then the complete bipartite graph $K_{m, k n}$ has an $S_{k}$-decomposition.

Proof. The proof is trivial.

Let $m$ and $n$ be positive integers. The disjoint union of graphs $G$ and $H$, denoted $G+H$, is the union of graphs $G$ and $H$ with disjoint vertex sets. The join of simple graphs $G$ and $H$, denoted $G \vee H$ is the graph obtained from the disjoint union $G+H$ by adding the edges $\{\{x, y\} \mid x \in V(G), y \in V(H)\}$. Also for any graph $G, m G$ is the graph consisting of $m$ pairwise disjoint copies of $G$. Furthermore, we denote the complete multigraph on $n$ vertices with multiplicity $m$ by $K_{n}^{m}[16]$.

Lemma 4. If $k$ is a positive integer and $s$ is a positive odd integer, then the graph $K_{s} \vee$ $\frac{(k-1)(s-1)}{2} K_{1}$ has an $S_{k}$-decomposition.

Proof. Let $k$ be a positive integer and $s$ be a positive odd integer. Consider the graph $K_{s} \vee \frac{(s-1)(k-1)}{2} K_{1}$. Label the vertices of $K_{s}$ with the elements of $\mathbb{Z}_{s}$ having subscript 1 and the remaining $\frac{(k-1)(s-1)}{2}$ vertices with the elements of $\mathbb{Z}_{\frac{(k-1)(s-1)}{2}}$ having the subscript 2 . Then, the following stars form a decomposition for $K_{s} \vee \frac{(k-1)(s-1)}{2} K_{1}$ with $k$-stars where $i \in \mathbb{Z}_{s}$ and $j=0,1, \ldots, \frac{s-3}{2}$ (see Figure 1).

$$
\left(i_{1} ;(i+j+1)_{1},((k-1) j)_{2},((k-1) j+1)_{2},((k-1) j+2)_{2}, \ldots,((k-1) j+k-2)_{2}\right)
$$




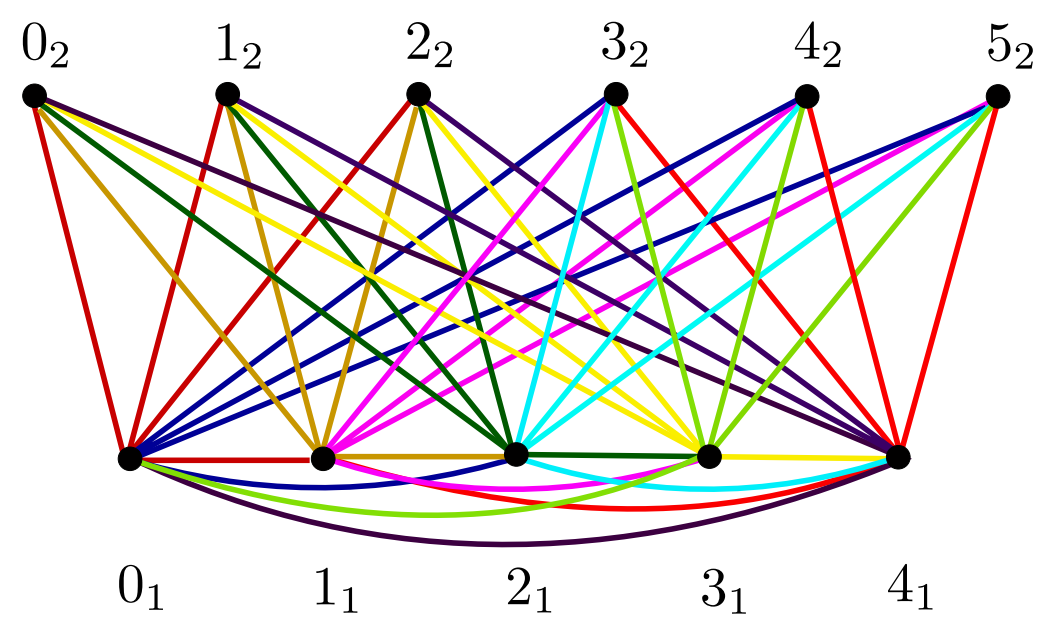

Figure 1: $S_{4}$-decomposition of $K_{5} \vee 6 K_{1}$

Lemma 5. If $k$ is a positive integer, $s$ is a positive odd integer, and $k \geq \frac{s-1}{2}$, then the graph $K_{s} \vee \frac{2 k-s+1}{2} K_{1}$ has an $S_{k}$-decomposition.

Proof. Let $k$ be a positive integer and $s$ be a positive odd integer such that $k \geq \frac{s-1}{2}$. Label the vertices of $K_{s}$ with the elements of $\mathbb{Z}_{s}$ having subscript 1 and the remaining $\frac{2 k-s+1}{2}$ vertices with the elements of $\mathbb{Z}_{\frac{2 k-s+1}{2}}$ having subscript 2. The following stars will form an $S_{k}$-decomposition for the graph $K_{s} \vee \frac{2 k-s+1}{2} K_{1}$ (see Figure 2).

$$
\left(i_{1} ;(i+1)_{1},(i+2)_{1}, \ldots,\left(i+\left(\frac{s-1}{2}\right)\right)_{1}, 0_{2}, 1_{2}, \ldots,\left(\frac{2 k-s-1}{2}\right)_{2}\right), i \in \mathbb{Z}_{s} .
$$

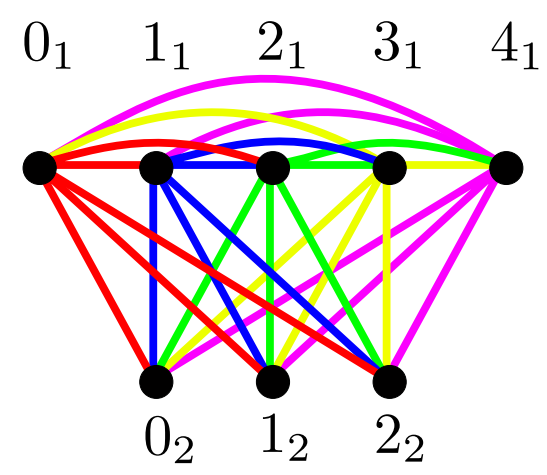

Figure 2: $S_{5}$-decomposition of $K_{5} \vee 3 K_{1}$

Corollary 6. If $k \geq 2$ is a positive integer, then the graph $K_{2 k-1}$ has a maximum packing with $k$-stars with a single edge as the leave graph.

Proof. For $k=2, K_{2 k-1}$ is a triangle and the result follows immediately. For $k>2$, write $K_{2 k-1}=K_{2 k-3} \vee K_{2}$. Letting $s=2 k-3$, the result follows by Lemma 5 . 
Corollary 7. If $n$ and $k$ are positive integers such that $n \equiv 2 k-1(\bmod 2 k)$, then $K_{n}$ has a maximum packing with $k$-stars with a single edge as the leave graph.

Proof. Let $n$ be a positive integer such that $n \equiv 2 k-1(\bmod 2 k)$. Write $K_{n}=K_{2 k-1} \vee$ $K_{n-2 k+1}$. Since $n \equiv 2 k-1(\bmod 2 k), K_{n-2 k+1}$ has an $S_{k}$-decomposition $R$ by Theorem 1 . Moreover, $K_{2 k-1}$ has a maximum packing $S$ with $k$-stars with a single edge as the leave, by Corollary 6 . Now, $K_{2 k-1, n-2 k+1}$ is a complete bipartite graph with one part of size a multiple of $k$ and hence, it has an $S_{k}$-decomposition $T$ by Lemma 3. Therefore, $R \cup S \cup T$ is a maximum packing of $K_{n}$ with $k$-stars with a single edge as the leave graph.

The following lemmas will greatly reduce the number of cases in the proofs of our main theorems.

Lemma 8. If $k$ is a positive odd integer, $n \geq \frac{k+1}{2}$ is an integer, and $H$ is the leave graph (excess graph) in an $S_{k}$-packing $\left(S_{k}\right.$-covering) of the complete graph $K_{n}$, then there exists an $S_{k}$-packing ( $S_{k}$-covering) of $K_{n+k}$ with $H$ as the leave graph (excess graph).

Proof. Let $k$ be a positive odd integer and $n \geq \frac{k+1}{2}$ be a positive integer. Write $K_{n+k}=$ $K_{k} \vee K_{n}$. Let $R$ be an $S_{k}$-packing of $K_{n}$ with the leave $H$. Label the vertices of $K_{n}$ with the elements of $\mathbb{Z}_{n}$ having subscript 1 and the vertices of $K_{k}$ with the elements of $\mathbb{Z}_{k}$ having subscript 2 . The vertices $0_{1}, 1_{1}, \ldots,\left(\frac{2 n-k-3}{2}\right)_{1}$, the vertices $0_{2}, 1_{2}, \ldots,(k-1)_{2}$, and the edges between these two sets of vertices form a complete bipartite graph with one part of size a multiple of $k$. Hence, by Lemma 3 , this complete bipartite graph has an $S_{k}$-decomposition, $S$. Moreover, the vertices $\left(\frac{2 n-k-1}{2}\right)_{1},\left(\frac{2 n-k+1}{2}\right)_{1}, \ldots,(n-1)_{1}$, the vertices $0_{2}, 1_{2}, \ldots,(k-1)_{2}$, the edges between these two sets, and the edges between the vertices of the second set form a $K_{k} \vee \frac{k+1}{2} K_{1}$. Hence, by Lemma 5 , the graph $K_{k} \vee \frac{k+1}{2} K_{1}$ has an $S_{k}$-decomposition, $T$. Therefore, $R \cup S \cup T$ forms an $S_{k}$-packing of $K_{n+k}$ with $H$ as the leave graph.

The proof is similar for the covering case.

Lemma 9. If $k$ and $n$ are positive integers such that $n \geq 2 k$, and $H$ is the leave graph (excess graph) in an $S_{k}$-packing $\left(S_{k}\right.$-covering) of the complete graph $K_{n}$, then there exists an $S_{k}$-packing $\left(S_{k}\right.$-covering) of $K_{n+2 k}$ with $H$ as the leave graph (excess graph).

Proof. Let $k$ and $n$ be positive integers such that $n \geq 2 k$. Write $K_{n+2 k}=K_{2 k} \vee K_{n}$. Let $R$ be an $S_{k}$-packing of $K_{n}$ with the leave $H$. The graph $K_{2 k}$ has an $S_{k}$-decomposition, $S$, by Theorem 1. Moreover, the vertices of $K_{n}$, the vertices of $K_{2 k}$, and the edges between these two sets form a complete bipartite graph with one part of size a multiple of $k$. Hence, this graph has an $S_{k}$-decomposition, $T$, by Lemma 3. Therefore, $R \cup S \cup T$ forms an $S_{k}$-packing of $K_{n+2 k}$ with the leave $H$.

The proof is similar for the covering case.

Note that the Lemmas 8 and 9 work for maximum packings and minimum coverings as particular cases, but also work for decompositions. 


\subsection{Packing and Covering the Complete Graph with 3-stars}

Theorem 10. Let $n \geq 5$ be an integer and the leave graph in a maximum packing of the complete graph $K_{n}$ with 3-stars have $i$ edges. For any graph $H$ with $i$ edges there exists a maximum packing of $K_{n}$ with 3-stars such that the leave graph is isomorphic to $H$.

Proof. By Lemma 8, it suffices to prove the theorem for $n=5,6$, and 7 . For $n=6$ or $7, K_{n}$ has an $S_{3}$-decomposition by Theorem 1 . For $n=5$, the leave has a single edge by Theorem 2. Therefore, the proof is complete.

Theorem 11. Let $n \geq 6$ be an integer and the excess graph in a minimum covering of the complete graph $K_{n}$ with 3-stars have $i$ edges. For any graph $H$ with $i$ edges there exists a minimum covering of $K_{n}$ with 3-stars such that the excess graph is isomorphic to $H$.

Proof. By Lemma 8, we only need to prove the theorem for $n=6,7$, and 8 . For $n=6$ or 7 , $K_{n}$ has an $S_{3}$-decomposition by Theorem 1 . For $n=8$, the excess has two edges by Theorem 2. Hence, the possible excesses are $S_{2}, 2 K_{2}$, and $K_{2}^{2}$. The excess $S_{2}$ is achieved by Roditty [10]. In order to obtain the excess $K_{2}^{2}$, write $K_{8}=K_{3} \vee K_{5}$. Label the vertices of $K_{5}$ with the elements of $\mathbb{Z}_{5}$ having subscript 1 and the vertices of $K_{3}$ with the elements of $\mathbb{Z}_{3}$ having subscript 2 . By Theorem $2, K_{5}$ has an $S_{3}$-packing, $R$, with a single edge, say $\left\{3_{1}, 4_{1}\right\}$ as the leave. Moreover, the following stars form a minimum $S_{3}$-covering, $S$, for the remaining graph with two multiple edges $\left\{0_{2}, 1_{2}\right\}$ as the excess.

$$
\begin{aligned}
& \left(0_{2} ; 1_{2}, 0_{1}, 1_{1}\right),\left(0_{2} ; 1_{2}, 2_{1}, 3_{1}\right),\left(1_{2} ; 0_{2}, 0_{1}, 1_{1}\right),\left(1_{2} ; 2_{2}, 2_{1}, 3_{1}\right) \\
& \left(2_{2} ; 0_{2}, 0_{1}, 1_{1}\right),\left(2_{2} ; 2_{1}, 3_{1}, 4_{1}\right),\left(4_{1} ; 3_{1}, 0_{2}, 1_{2}\right)
\end{aligned}
$$

Therefore, $R \cup S$ forms a minimum covering for $K_{8}$ with 3 -stars with the excess $K_{2}^{2}$. Now, substituting the stars $\left(0_{2} ; 0_{1}, 1_{1}, 2_{1}\right)$ and $\left(1_{2} ; 0_{1}, 1_{1}, 3_{1}\right)$ for $\left(0_{2} ; 1_{2}, 0_{1}, 1_{1}\right)$ and $\left(1_{2} ; 0_{2}, 0_{1}, 1_{1}\right)$ respectively in $R \cup S$ will result in a minimum covering of $K_{8}$ with the excess $2 K_{2}$.

\subsection{Packing and Covering the Complete Graph with 4-stars}

Theorem 12 ([6]). Let $n \geq 7$ be an integer and the leave graph in a maximum packing of the complete graph $K_{n}$ with 4-stars have $i$ edges. For any graph $H$ with $i$ edges there exists a maximum packing of $K_{n}$ with 4-stars such that the leave graph is isomorphic to $H$.

Proof. By Lemma 9, it suffices to prove the theorem for $n=7,8,9, \ldots, 14$. The proofs of these cases are included in the general proofs given in [6].

Theorem $13([6])$. Let $n \geq 8$ be an integer and the excess graph in a minimum covering of the complete graph $K_{n}$ with 4-stars have $i$ edges. For any graph $H$ with $i$ edges there exists a minimum covering of $K_{n}$ with 4 -stars such that the excess graph is isomorphic to $H$.

Proof. Again by Lemma 9, we only need to prove the theorem for $n=8,9,10, \ldots, 15$. The proofs of these cases are included in the general proofs given in [6] as well. 


\section{Main Results}

\subsection{All Possible Leave Graphs}

In 1986 Roditty solved the problem of packing the complete graph $K_{n}$ with 5-stars [12]. We prove that we can achieve all the non-isomorphic possible leave graphs, thus solving the spectrum problem.

Theorem 14. Let $n \geq 9$ be an integer and the leave graph in a maximum packing of the complete graph $K_{n}$ with 5-stars have $i$ edges. For any graph $H$ with $i$ edges there exists a maximum packing of $K_{n}$ with 5-stars such that the leave graph is isomorphic to $H$.

Proof. The complete graph $K_{n}$ has an $S_{5}$-decomposition for $n \equiv 0,1,5$, or $6(\bmod 10)$ by Theorem 1. We show that for the remaining cases we have maximum packings with all the possible leave graphs.

By Corollary 7 and Lemma 8, the proof is complete for $n \equiv 4$ and 9 (mod 10). Now, by Lemma 8, we only need to prove the theorem for the cases when $n \equiv 2$ and $3(\bmod 10)$. Again by Lemma 8, it suffices to prove the theorem for $n=12$ and $n=13$.

Case 1. $n=12$

Write $K_{12}=K_{10} \vee K_{2}$. Label the vertices of $K_{10}$ with the elements of $\mathbb{Z}_{10}$ having subscript 1 and the vertices of $K_{2}$ with the elements of $\mathbb{Z}_{2}$ having subscript 2 . By Theorem 1 , $K_{10}$ has an $S_{5}$-decomposition, $R$. Now, the vertices $0_{1}, 1_{1}, \ldots, 9_{1}$, the vertices $0_{2}, 1_{2}$, and the edges between these two sets of vertices form a complete bipartite graph with one part of size a multiple of 5 . Hence, by Lemma 3 , this complete bipartite graph has an $S_{5}$-decomposition, $S$. Now, $R \cup S$ forms a maximum packing of $K_{12}$ with 5 -stars with the single edge $\left\{0_{2}, 1_{2}\right\}$ as the leave graph.

Case 2. $n=13$

For this case, the leave graph has 3 edges by Theorem 2 . Hence, the possible leaves are $K_{3}, S_{3}, P_{4}, 3 K_{2}$, and $S_{2}+K_{2}$. In order to obtain $K_{3}$, write $K_{13}=K_{10} \vee K_{3}$. The graph $K_{10}$ has an $S_{5}$-decomposition, $R$, by Theorem 1. Moreover, 10 is a multiple of 5 and hence, by Lemma $3, K_{3,10}$ has an $S_{5}$-decomposition, $S$. Therefore, $R \cup S$ forms a maximum packing of $K_{13}$ with 5 -stars with a $K_{3}$ as the leave graph.

In order to obtain $S_{3}$ as the leave, again write $K_{13}=K_{10} \vee K_{3}$. Then $K_{10}$ has an $S_{5^{-}}$ decomposition, $R^{\prime}$, by Theorem 1 . Label the vertices of $K_{10}$ with the elements of $\mathbb{Z}_{10}$ with subscript 1 and the vertices of $K_{3}$ with the elements of $\mathbb{Z}_{3}$ with subscript 2 . The vertices $0_{1}, 1_{1}, 2_{1}, 3_{1}$, the vertices $0_{2}, 1_{2}, 2_{2}$, the edges between these two sets of vertices, and the edges between the vertices of the latter set will form a $K_{3} \vee 4 K_{1}$. By Lemma $4, K_{3} \vee 4 K_{1}$ has an $S_{5^{-}}$ decomposition, $S^{\prime}$. Let $S^{\prime}$ be formed by the stars $\left(0_{2} ; 1_{2}, 0_{1}, 1_{1}, 2_{1}, 3_{1}\right),\left(1_{2} ; 2_{2}, 0_{1}, 1_{1}, 2_{1}, 3_{1}\right)$, and $\left(2_{2} ; 0_{2}, 0_{1}, 1_{1}, 2_{1}, 3_{1}\right)$. Now, the vertices $4_{1}, 5_{1}, 6_{1}, 7_{1}, 8_{1}$, the vertices $0_{2}, 1_{2}, 2_{2}$, and the edges between these two sets of vertices form a complete bipartite graph with one part of 
size a multiple of 5 . Hence, by Lemma 3 , this graph has an $S_{5}$-decomposition, $T^{\prime}$. Let $T^{\prime}$ be formed by the stars $\left(0_{2} ; 4_{1}, 5_{1}, 6_{1}, 7_{1}, 8_{1}\right),\left(1_{2} ; 4_{1}, 5_{1}, 6_{1}, 7_{1}, 8_{1}\right)$, and $\left(2_{2} ; 4_{1}, 5_{1}, 6_{1}, 7_{1}, 8_{1}\right)$. Therefore, $R^{\prime} \cup S^{\prime} \cup T^{\prime}$ forms a maximum packing for $K_{13}$ with the 3 -star $\left(9_{1} ; 0_{2}, 1_{2}, 2_{2}\right)$ as the leave graph.

Substituting the star $\left(0_{2} ; 4_{1}, 5_{1}, 6_{1}, 7_{1}, 9_{1}\right)$ for $\left(0_{2} ; 4_{1}, 5_{1}, 6_{1}, 7_{1}, 8_{1}\right)$ in the packing $R^{\prime} \cup S^{\prime} \cup T^{\prime}$ gives us a maximum packing $U$ of $K_{13}$ with $S_{2}+K_{2}$ as the leave graph.

Substituting the star $\left(1_{2} ; 2_{2}, 0_{1}, 1_{1}, 2_{1}, 9_{1}\right)$ for $\left(1_{2} ; 2_{2}, 0_{1}, 1_{1}, 2_{1}, 3_{1}\right)$ in the packing $U$ results in a maximum packing for $K_{13}$ with $3 K_{2}$ as the leave graph.

Finally, considering the packing $R^{\prime} \cup S^{\prime} \cup T^{\prime}$ and substituting the star $\left(0_{2} ; 0_{1}, 1_{1}, 2_{1}, 3_{1}, 9_{1}\right)$ for $\left(0_{2} ; 1_{2}, 0_{1}, 1_{1}, 2_{1}, 3_{1}\right)$ gives us a maximum packing for $K_{13}$ with the leave $P_{4}$. This completes the proof in this case.

\subsection{All Possible Excess Graphs}

In the previous subsection, we showed how to achieve all possible leave graphs in packing the complete graph with 5-stars. Now, we prove that all possible excess graphs in covering the complete graph with 5-stars are also achievable. Refer to Table 4 at the end of this section for all possible leaves and excesses in different congruence classes.

Theorem 15. Let $n \geq 10$ be an integer let the excess graph in a minimum covering of the complete graph $K_{n}$ with 5-stars have $i$ edges. For any graph $H$ with $i$ edges there exists a minimum covering of $K_{n}$ with 5-stars such that the excess graph is isomorphic to $H$, except for the excess $K_{2}^{4}$ which is not achievable for $n=12$.

Proof. The complete graph $K_{n}$ has an $S_{5}$-decomposition for $n \equiv 0,1,5$, or $6(\bmod 10)$ by Theorem 1. We show that for the remaining cases we have minimum coverings with all the possible excess graphs.

By Lemma 8, we only need to prove the theorem for the cases when $n \equiv 2,3$, and $4(\bmod 10)$. Also by Lemma 8, it suffices to consider the cases $n=12, n=13$, and $n=14$.

Case 1. $n=12$. By Theorem 2, the excess graph has 4 edges in this case. Figure 3 shows all possible excesses with 4 edges $\left(E_{i}\right.$ demonstrates the $i$ th excess). Let $P$ be a maximum packing of $K_{12}$ with 5 -stars. Since the leave graph in a maximum $S_{5}$-packing is a single edge, if we add a 5 -star including that single edge, we obtain $E_{1}$ as the excess.

In order to achieve $E_{14}$, write $K_{12}=K_{3} \vee K_{9}$. Label the vertices of $K_{9}$ with the elements of $\mathbb{Z}_{9}$ having subscript 1 and the vertices of $K_{3}$ with the elements of $\mathbb{Z}_{3}$ having subscript 2. The following stars form a maximum $S_{5}$-packing, $R$, for $K_{9}$ with the single edge $\left\{7_{1}, 8_{1}\right\}$ as the leave.

$$
\begin{aligned}
& \left(0_{1} ; 1_{1}, 2_{1}, 3_{1}, 7_{1}, 8_{1}\right),\left(1_{1} ; 2_{1}, 3_{1}, 4_{1}, 7_{1}, 8_{1}\right),\left(2_{1} ; 3_{1}, 4_{1}, 5_{1}, 7_{1}, 8_{1}\right),\left(3_{1} ; 4_{1}, 5_{1}, 6_{1}, 7_{1}, 8_{1}\right), \\
& \left(4_{1} ; 5_{1}, 6_{1}, 0_{1}, 7_{1}, 8_{1}\right),\left(5_{1} ; 6_{1}, 0_{1}, 1_{1}, 7_{1}, 8_{1}\right),\left(6_{1} ; 0_{1}, 1_{1}, 2_{1}, 7_{1}, 8_{1}\right) .
\end{aligned}
$$



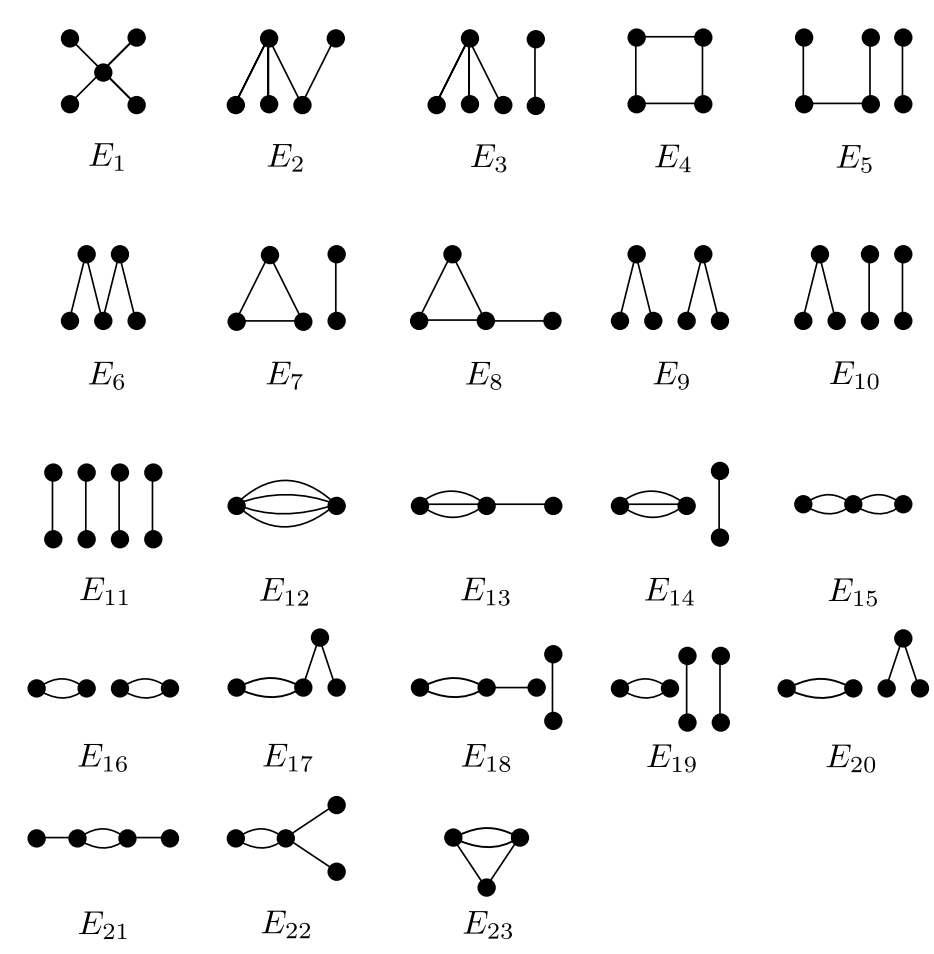

Figure 3: All possible 4-edge excesses

Moreover, the following stars form a minimum $S_{5}$-covering, $S$, of the remaining edges with three multiple edges $\left\{0_{2}, 1_{2}\right\}$ and the edge $\left\{6_{1}, 8_{1}\right\}$ as the excess, which forms a graph isomorphic to $E_{14}$.

$$
\begin{aligned}
& \left(0_{2} ; 1_{2}, 0_{1}, 1_{1}, 2_{1}, 3_{1}\right),\left(1_{2} ; 0_{2}, 0_{1}, 1_{1}, 2_{1}, 3_{1}\right),\left(0_{2} ; 1_{2}, 4_{1}, 5_{1}, 6_{1}, 7_{1}\right),\left(1_{2} ; 0_{2}, 4_{1}, 5_{1}, 6_{1}, 7_{1}\right), \\
& \left(2_{2} ; 0_{2}, 0_{1}, 1_{1}, 2_{1}, 3_{1}\right),\left(2_{2} ; 1_{2}, 4_{1}, 5_{1}, 6_{1}, 7_{1}\right),\left(8_{1} ; 6_{1}, 7_{1}, 0_{2}, 1_{2}, 2_{2}\right) .
\end{aligned}
$$

Therefore, $R \cup S$ forms a minimum $S_{5}$-covering for $K_{12}$ with the excess $E_{14}$ (see Figure 4, where each thick line demonstrates a 4 -star). Substituting the stars $\left(0_{1} ; 1_{1}, 2_{1}, 3_{1}, 7_{1}, 0_{2}\right)$ and

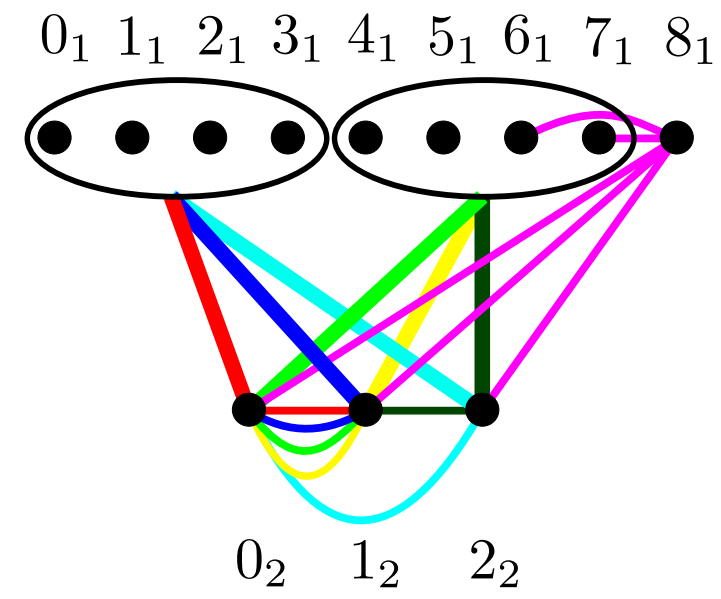

Figure 4: Minimum covering of $K_{12}$ with the excess $E_{14}$ 
$\left(8_{1} ; 0_{1}, 7_{1}, 0_{2}, 1_{2}, 2_{2}\right)$ for $\left(0_{1} ; 1_{1}, 2_{1}, 3_{1}, 7_{1}, 8_{1}\right)$ and $\left(8_{1} ; 6_{1}, 7_{1}, 0_{2}, 1_{2}, 2_{2}\right)$ respectively, leads to a minimum $S_{5}$-covering, $U$, with the excess $E_{13}$.

Now, we obtain all possible excesses by substitution of some stars with some other ones in the coverings $R \cup S$ and $U$. The substitutions are given in Tables 1 and 2 .

\begin{tabular}{|c|c|c|}
\hline New Star(s) & Previous Star(s) & Excess \\
\hline$\left(0_{2} ; 6_{1}, 0_{1}, 1_{1}, 2_{1}, 3_{1}\right)$ and $\left(0_{2} ; 0_{1}, 4_{1}, 5_{1}, 6_{1}, 7_{1}\right)$ & $\left(0_{2} ; 1_{2}, 0_{1}, 1_{1}, 2_{1}, 3_{1}\right)$ and $\left(0_{2} ; 1_{2}, 4_{1}, 5_{1}, 6_{1}, 7_{1}\right)$ & $E_{2}$ \\
\hline$\left(0_{2} ; 4_{1}, 0_{1}, 1_{1}, 2_{1}, 3_{1}\right)$ and $\left(0_{2} ; 0_{1}, 4_{1}, 5_{1}, 6_{1}, 7_{1}\right)$ & $\left(0_{2} ; 1_{2}, 0_{1}, 1_{1}, 2_{1}, 3_{1}\right)$ and $\left(0_{2} ; 1_{2}, 4_{1}, 5_{1}, 6_{1}, 7_{1}\right)$ & $E_{3}$ \\
\hline$\left(0_{2} ; 6_{1}, 0_{1}, 1_{1}, 2_{1}, 3_{1}\right)$ and $\left(1_{2} ; 8_{1}, 0_{1}, 1_{1}, 2_{1}, 3_{1}\right)$ & $\left(0_{2} ; 1_{2}, 0_{1}, 1_{1}, 2_{1}, 3_{1}\right)$ and $\left(1_{2} ; 0_{2}, 0_{1}, 1_{1}, 2_{1}, 3_{1}\right)$ & $E_{4}$ \\
\hline$\left(0_{2} ; 4_{1}, 0_{1}, 1_{1}, 2_{1}, 3_{1}\right)$ and $\left(1_{2} ; 5_{1}, 0_{1}, 1_{1}, 2_{1}, 3_{1}\right)$ & $\left(0_{2} ; 1_{2}, 0_{1}, 1_{1}, 2_{1}, 3_{1}\right)$ and $\left(1_{2} ; 0_{2}, 0_{1}, 1_{1}, 2_{1}, 3_{1}\right)$ & $E_{5}$ \\
\hline$\left(0_{2} ; 6_{1}, 0_{1}, 1_{1}, 2_{1}, 3_{1}\right)$ and $\left(1_{2} ; 5_{1}, 0_{1}, 1_{1}, 2_{1}, 3_{1}\right)$ & $\left(0_{2} ; 1_{2}, 0_{1}, 1_{1}, 2_{1}, 3_{1}\right)$ and $\left(1_{2} ; 0_{2}, 0_{1}, 1_{1}, 2_{1}, 3_{1}\right)$ & $E_{6}$ \\
\hline$\left(1_{2} ; 2_{2}, 0_{1}, 1_{1}, 2_{1}, 3_{1}\right)$ and $\left(0_{2} ; 2_{2}, 4_{1}, 5_{1}, 6_{1}, 7_{1}\right)$ & $\left(1_{2} ; 0_{2}, 0_{1}, 1_{1}, 2_{1}, 3_{1}\right)$ and $\left(0_{2} ; 1_{2}, 4_{1}, 5_{1}, 6_{1}, 7_{1}\right)$ & $E_{7}$ \\
\hline $\begin{array}{c}\left(0_{2} ; 4_{1}, 0_{1}, 1_{1}, 2_{1}, 3_{1}\right),\left(1_{2} ; 5_{1}, 0_{1}, 1_{1}, 2_{1}, 3_{1}\right), \\
\text { and }\left(1_{2} ; 2_{2}, 4_{1}, 5_{1}, 6_{1}, 7_{1}\right)\end{array}$ & $\begin{array}{c}\left(0_{2} ; 1_{2}, 0_{1}, 1_{1}, 2_{1}, 3_{1}\right),\left(1_{2} ; 0_{2}, 0_{1}, 1_{1}, 2_{1}, 3_{1}\right), \\
\text { and }\left(1_{2} ; 0_{2}, 4_{1}, 5_{1}, 6_{1}, 7_{1}\right)\end{array}$ & $E_{10}$ \\
\hline $\begin{array}{c}\left(0_{2} ; 4_{1}, 0_{1}, 1_{1}, 2_{1}, 3_{1}\right),\left(1_{2} ; 5_{1}, 0_{1}, 1_{1}, 2_{1}, 3_{1}\right) \\
\left(1_{2} ; 2_{2}, 4_{1}, 5_{1}, 6_{1}, 7_{1}\right), \text { and }\left(2_{2} ; 3_{1}, 4_{1}, 5_{1}, 6_{1}, 7_{1}\right)\end{array}$ & $\begin{array}{c}\left(0_{2} ; 1_{2}, 0_{1}, 1_{1}, 2_{1}, 3_{1}\right),\left(1_{2} ; 0_{2}, 0_{1}, 1_{1}, 2_{1}, 3_{1}\right), \\
\left(1_{2} ; 0_{2}, 4_{1}, 5_{1}, 6_{1}, 7_{1}\right), \text { and }\left(2_{2} ; 1_{2}, 4_{1}, 5_{1}, 6_{1}, 7_{1}\right)\end{array}$ & $E_{11}$ \\
\hline$\left(0_{2} ; 8_{1}, 4_{1}, 5_{1}, 6_{1}, 7_{1}\right)$ & $\left(0_{2} ; 1_{2}, 4_{1}, 5_{1}, 6_{1}, 7_{1}\right)$ & $E_{17}$ \\
\hline$\left(0_{2} ; 2_{1}, 4_{1}, 5_{1}, 6_{1}, 7_{1}\right)$ & $\left(0_{2} ; 1_{2}, 4_{1}, 5_{1}, 6_{1}, 7_{1}\right)$ & $E_{18}$ \\
\hline$\left(1_{2} ; 2_{2}, 4_{1}, 5_{1}, 6_{1}, 7_{1}\right)$ and $\left(2_{2} ; 3_{1}, 4_{1}, 5_{1}, 66_{1}, 7_{1}\right)$ & $\left(1_{2} ; 0_{2}, 4_{1}, 5_{1}, 6_{1}, 7_{1}\right)$ and $\left(2_{2} ; 1_{2}, 4_{1}, 5_{1}, 6_{1}, 7_{1}\right)$ & $E_{19}$ \\
\hline$\left(0_{2} ; 2_{2}, 0_{1}, 1_{1}, 2_{1}, 3_{1}\right)$ and $\left(2_{2} ; 6_{1}, 0_{1}, 1_{1}, 2_{1}, 3_{1}\right)$ & $\left(0_{2} ; 1_{2}, 0_{1}, 1_{1}, 2_{1}, 3_{1}\right)$ and $\left(2_{2} ; 0_{2}, 0_{1}, 1_{1}, 2_{1}, 3_{1}\right)$ & $E_{20}$ \\
\hline
\end{tabular}

Table 1: Substitutions in the covering $R \cup S$ to obtain different excesses for $n=12$

\begin{tabular}{ccc}
\hline \hline New Star $(\mathrm{s})$ & Previous Star $(\mathrm{s})$ & Excess Number \\
\hline$\left(1_{2} ; 2_{2}, 0_{1}, 1_{1}, 2_{1}, 3_{1}\right)$ and $\left(0_{2} ; 2_{2}, 4_{1}, 5_{1}, 6_{1}, 7_{1}\right)$ & $\left(1_{2} ; 0_{2}, 0_{1}, 1_{1}, 2_{1}, 3_{1}\right)$ and $\left(0_{2} ; 1_{2}, 4_{1}, 5_{1}, 6_{1}, 7_{1}\right)$ & $E_{8}$ \\
\hline$\left(0_{2} ; 4_{1}, 0_{1}, 1_{1}, 2_{1}, 3_{1}\right),\left(1_{2} ; 2_{1}, 4_{1}, 5_{1}, 6_{1}, 7_{1}\right)$, & $\left(0_{2} ; 1_{2}, 0_{1}, 1_{1}, 2_{1}, 3_{1}\right),\left(1_{2} ; 0_{2}, 4_{1}, 5_{1}, 6_{1}, 7_{1}\right)$, & \\
and $\left(1_{2} ; 5_{1}, 0_{1}, 1_{1}, 2_{1}, 3_{1}\right)$ & and $\left(1_{2} ; 0_{2}, 0_{1}, 1_{1}, 2_{1}, 3_{1}\right)$ & $E_{9}$ \\
\hline$\left(0_{2} ; 0_{1}, 4_{1}, 5_{1}, 6_{1}, 7_{1}\right)$ & $\left(0_{2} ; 1_{2}, 4_{1}, 5_{1}, 6_{1}, 7_{1}\right)$ & $E_{15}$ \\
\hline$\left(0_{2} ; 0_{1}, 4_{1}, 5_{1}, 6_{1}, 7_{1}\right),\left(1_{2} ; 8_{1}, 0_{1}, 1_{1}, 2_{1}, 3_{1}\right)$, & $\left(0_{2} ; 1_{2}, 4_{1}, 5_{1}, 6_{1}, 7_{1}\right),\left(1_{2} ; 0_{2}, 0_{1}, 1_{1}, 2_{1}, 3_{1}\right)$, & \\
and $\left(1_{2} ; 8_{1}, 4_{1}, 5_{1}, 6_{1}, 7_{1}\right)$ & and $\left(1_{2} ; 0_{2}, 4_{1}, 5_{1}, 6_{1}, 7_{1}\right)$ & $E_{16}$ \\
\hline$\left(1_{2} ; 4_{1}, 0_{1}, 1_{1}, 2_{1}, 3_{1}\right)$ & $\left(1_{2} ; 0_{2}, 0_{1}, 1_{1}, 2_{1}, 3_{1}\right)$ & $E_{21}$ \\
\hline$\left(0_{2} ; 1_{1}, 4_{1}, 5_{1}, 6_{1}, 7_{1}\right)$ & $\left(0_{2} ; 1_{2}, 4_{1}, 5_{1}, 6_{1}, 7_{1}\right)$ & $E_{22}$ \\
\hline$\left(1_{2} ; 0_{1}, 4_{1}, 5_{1}, 6_{1}, 7_{1}\right)$ & $\left(1_{2} ; 0_{2}, 4_{1}, 5_{1}, 6_{1}, 7_{1}\right)$ & $E_{23}$ \\
\hline
\end{tabular}

Table 2: Substitutions in the covering $U$ to obtain different excesses for $n=12$

Now, we prove that for $n=12$, the excess graph $E_{12}=K_{2}^{4}$ is not achievable. Assume to the contrary that $Q$ is a minimum covering of $K_{12}$ with 5 -stars with the excess $E_{12}$. Let $x$ and $y$ be the end vertices of the four multiple edges of the excess. Since the four multiple edges form the excess, $Q$ contains five multiple edges $\{x, y\}$. We claim that each of the vertices $x$ and $y$ can be at most the center of two stars of $Q$ containing the edge $\{x, y\}$. Assume to the contrary that $x$ is the center of three such stars. Hence, there exist at least three disjoint sets of four vertices other than $x$ and $y$. This contradicts $n=12$ and hence, our claim is true. Therefore, there are at most four multiple edges between $x$ and $y$ in $Q$, which shows there is no minimum covering of $K_{12}$ with 5 -stars with the excess $E_{12}$.

Case 2. $n=13$. 
In this case, the excess graph has 2 edges by Theorem 2. Hence, the possible excesses are $S_{2}, 2 K_{2}$, and $K_{2}^{2}$.

The excess $S_{2}$ is easily achievable by adding a 5 -star to a maximum packing of $K_{13}$ with the leave $S_{3}$.

In order to obtain the excess $2 K_{2}$, write $K_{13}=K_{9} \vee K_{4}$. Label the vertices of $K_{9}$ with the elements of $\mathbb{Z}_{9}$ having subscript 1 , the vertices of $K_{4}$ with the elements of $\mathbb{Z}_{4}$ having subscript 2. Let $R$ be a maximum packing of $K_{9}$ with the single edge $\left\{7_{1}, 8_{1}\right\}$ as the leave. Consider the vertices $0_{1}, 1_{1}, 2_{1}, 3_{1}$, and $4_{1}$, the vertices $0_{2}, 1_{2}, 2_{2}$, and $3_{2}$, and the edges between these two sets form a complete bipartite graph with one part of size a multiple of 5 . Hence, by Lemma 3, this bipartite graph has an $S_{5}$-decomposition, $S$. Furthermore, the following stars form a minimum covering, $T$, for the remaining graph with the edges $\left\{0_{2}, 2_{2}\right\}$ and $\left\{1_{2}, 3_{2}\right\}$ as the excess.

$$
\begin{aligned}
& \left(0_{2} ; 1_{2}, 2_{2}, 5_{1}, 6_{1}, 7_{1}\right),\left(1_{2} ; 2_{2}, 3_{2}, 5_{1}, 6_{1}, 7_{1}\right),\left(2_{2} ; 3_{2}, 0_{2}, 5_{1}, 6_{1}, 7_{1}\right), \\
& \left(3_{2} ; 0_{2}, 1_{2}, 5_{1}, 6_{1}, 7_{1}\right),\left(8_{1} ; 7_{1}, 0_{2}, 1_{2}, 2_{2}, 3_{2}\right) .
\end{aligned}
$$

Therefore, $R \cup S \cup T$ forms a minimum covering of $K_{13}$ with the excess $2 K_{2}$ (see Figure 5 ). In order to achieve the excess $K_{2}^{2}$, partition and label the vertices of $K_{13}$ as before and let

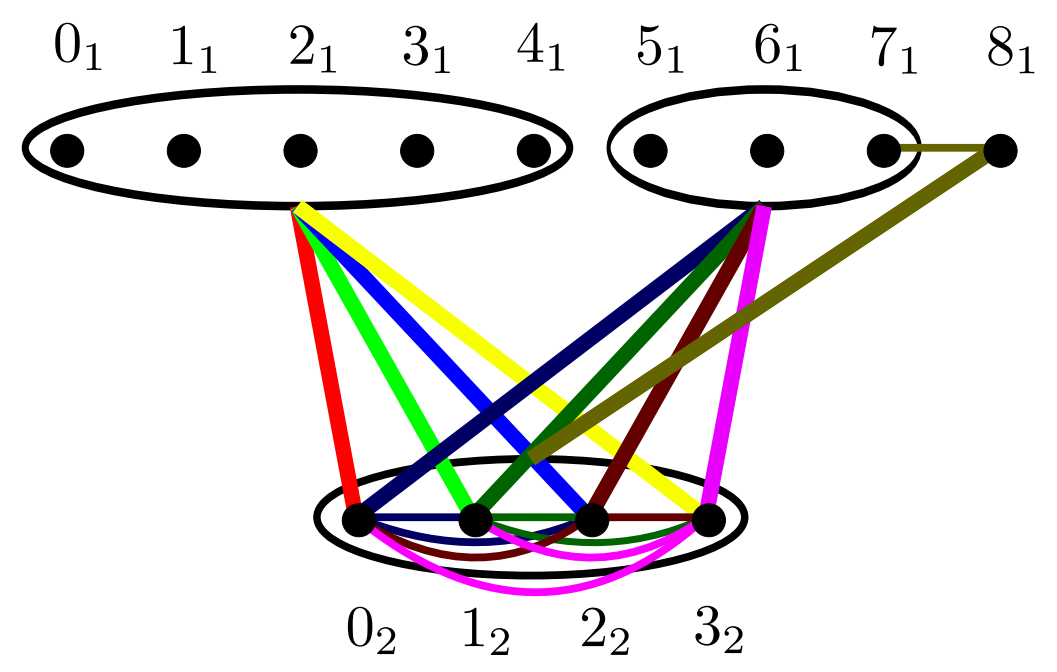

Figure 5: Minimum covering of $K_{13}$ with the excess $2 K_{2}$

$R$ be the same packing of $K_{9}$ with the same edge as the leave. The following stars form a minimum covering, $R^{\prime \prime}$, of the remaining graph with two multiple edges $\left\{0_{2}, 1_{2}\right\}$ as the excess.

$$
\begin{aligned}
& \left(0_{2} ; 1_{2}, 0_{1}, 1_{1}, 2_{1}, 3_{1}\right),\left(0_{2} ; 1_{2}, 4_{1}, 5_{1}, 6_{1}, 7_{1}\right),\left(1_{2} ; 0_{2}, 0_{1}, 1_{1}, 2_{1}, 3_{1}\right),\left(1_{2} ; 2_{2}, 4_{1}, 5_{1}, 6_{1}, 7_{1}\right), \\
& \left(2_{2} ; 0_{2}, 0_{1}, 1_{1}, 2_{1}, 3_{1}\right),\left(2_{2} ; 3_{2}, 4_{1}, 5_{1}, 6_{1}, 7_{1}\right),\left(3_{2} ; 0_{2}, 0_{1}, 1_{1}, 2_{1}, 3_{1}\right),\left(3_{2} ; 1_{2}, 4_{1}, 5_{1}, 6_{1}, 7_{1}\right), \\
& \left(8_{1} ; 7_{1}, 0_{2}, 1_{2}, 2_{2}, 3_{2}\right) .
\end{aligned}
$$




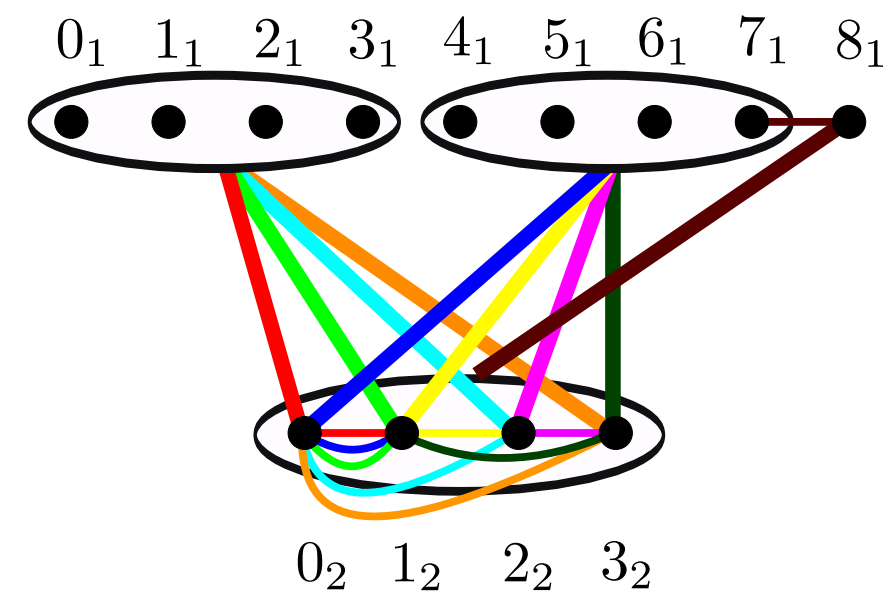

Figure 6: Minimum covering of $K_{13}$ with the excess $K_{2}^{2}$

Therefore, $R \cup R^{\prime \prime}$ forms a minimum covering for $K_{13}$ with the excess $K_{2}^{2}$ (see Figure 6 ). Case 3. $n=14$

In this case, the excess graph has 4 edges by Theorem 2. Hence, the possible excesses are the ones shown in Figure 3. Again, since the leave graph in a maximum $S_{5}$-packing is a single edge, if we add a 5 -star including that single edge, we obtain $E_{1}$ as the excess.

In order to achieve the excess $E_{12}$, write $K_{14}=K_{5} \vee K_{9}$. Label the vertices of $K_{9}$ with the elements of $\mathbb{Z}_{9}$ having subscript 1 and the vertices of $K_{5}$ with the elements of $\mathbb{Z}_{5}$ having subscript 2 . Let $R$ be a maximum packing of $K_{9}$ with 5 -stars and let the leave be the edge $\left\{7_{1}, 8_{1}\right\}$. The following stars form a minimum covering, $S$, of the remaining edges with four multiple edges on the vertices $0_{2}$ and $1_{2}$ as the excess graph.

$$
\begin{aligned}
& \left(0_{2} ; 1_{2}, 0_{1}, 1_{1}, 2_{1}, 3_{1}\right),\left(0_{2} ; 1_{2}, 4_{1}, 5_{1}, 6_{1}, 7_{1}\right),\left(1_{2} ; 0_{2}, 0_{1}, 1_{1}, 2_{1}, 3_{1}\right),\left(1_{2} ; 0_{2}, 4_{1}, 5_{1}, 6_{1}, 7_{1}\right), \\
& \left(0_{2} ; 1_{2}, 2_{2}, 3_{2}, 4_{2}, 8_{1}\right),\left(2_{2} ; 1_{2}, 0_{1}, 1_{1}, 2_{1}, 3_{1}\right),\left(2_{2} ; 3_{2}, 4_{1}, 5_{1}, 6_{1}, 7_{1}\right),\left(3_{2} ; 4_{2}, 0_{1}, 1_{1}, 2_{1}, 3_{1}\right), \\
& \left(3_{2} ; 1_{2}, 4_{1}, 5_{1}, 6_{1}, 7_{1}\right),\left(4_{2} ; 1_{2}, 0_{1}, 1_{1}, 2_{1}, 3_{1}\right),\left(4_{2} ; 2_{2}, 4_{1}, 5_{1}, 6_{1}, 7_{1}\right),\left(8_{1} ; 7_{1}, 1_{2}, 2_{2}, 3_{2}, 4_{2}\right) .
\end{aligned}
$$

Therefore, $R \cup S$ forms a minimum covering of $K_{14}$ with the excess $E_{12}$ (see Figure 7 ). Consider the covering $R \cup S$. Table 3 shows the star substitutions in $R \cup S$ needed to achieve each excess other than excesses 11 and 12. In the following table, the first, second, and third column demonstrate the new star(s), the previous star(s), and the excess number respectively. 


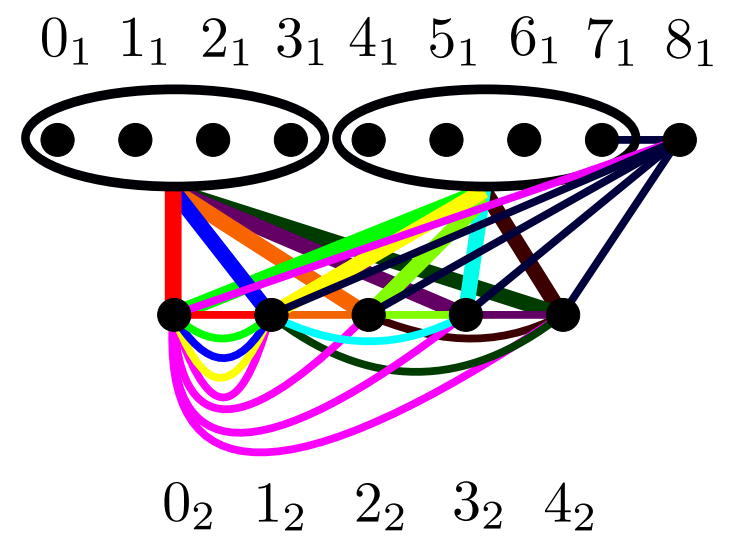

Figure 7: Minimum covering of $K_{14}$ with the excess $E_{12}$

\begin{tabular}{|c|c|c|}
\hline New $\operatorname{Star}(\mathrm{s})$ & Previous Star(s) & Excess \\
\hline $\begin{array}{c}\left(0_{2} ; 0_{1}, 4_{1}, 5_{1}, 6_{1}, 7_{1}\right),\left(0_{2} ; 4_{1}, 0_{1}, 1_{1}, 2_{1}, 3_{1}\right), \\
\text { and }\left(1_{2} ; 2_{2}, 4_{1}, 5_{1}, 6_{1}, 7_{1}\right)\end{array}$ & $\begin{array}{c}\left(0_{2} ; 1_{2}, 4_{1}, 5_{1}, 6_{1}, 7_{1}\right),\left(0_{2} ; 1_{2}, 0_{1}, 1_{1}, 2_{1}, 3_{1}\right), \\
\text { and }\left(1_{2} ; 0_{2}, 4_{1}, 5_{1}, 6_{1}, 7_{1}\right)\end{array}$ & $E_{2}$ \\
\hline $\begin{array}{c}\left(0_{2} ; 0_{1}, 4_{1}, 5_{1}, 6_{1}, 7_{1}\right),\left(0_{2} ; 4_{1}, 0_{1}, 1_{1}, 2_{1}, 3_{1}\right) \\
\left(1_{2} ; 2_{2}, 4_{1}, 5_{1}, 6_{1}, 7_{1}\right), \text { and }\left(2_{2} ; 5_{1}, 0_{1}, 1_{1}, 2_{1}, 3_{1}\right)\end{array}$ & $\begin{array}{c}\left(0_{2} ; 1_{2}, 4_{1}, 5_{1}, 6_{1}, 7_{1}\right),\left(0_{2} ; 1_{2}, 0_{1}, 1_{1}, 2_{1}, 3_{1}\right) \\
\left(1_{2} ; 0_{2}, 4_{1}, 5_{1}, 6_{1}, 7_{1}\right), \text { and }\left(2_{2} ; 1_{2}, 0_{1}, 1_{1}, 2_{1}, 3_{1}\right)\end{array}$ & $E_{3}$ \\
\hline $\begin{array}{c}\left(0_{2} ; 0_{1}, 4_{1}, 5_{1}, 6_{1}, 7_{1}\right),\left(0_{2} ; 4_{1}, 0_{1}, 1_{1}, 2_{1}, 3_{1}\right) \\
\left(1_{2} ; 4_{1}, 0_{1}, 1_{1}, 2_{1}, 3_{1}\right), \text { and }\left(1_{2} ; 0_{1}, 4_{1}, 5_{1}, 6_{1}, 7_{1}\right)\end{array}$ & $\begin{array}{c}\left(0_{2} ; 1_{2}, 4_{1}, 5_{1}, 6_{1}, 7_{1}\right),\left(0_{2} ; 1_{2}, 0_{1}, 1_{1}, 2_{1}, 3_{1}\right), \\
\left(1_{2} ; 0_{2}, 0_{1}, 1_{1}, 2_{1}, 3_{1}\right), \text { and }\left(1_{2} ; 0_{2}, 4_{1}, 5_{1}, 6_{1}, 7_{1}\right)\end{array}$ & $E_{4}$ \\
\hline $\begin{array}{c}\left(0_{2} ; 0_{1}, 4_{1}, 5_{1}, 6_{1}, 7_{1}\right),\left(0_{2} ; 4_{1}, 0_{1}, 1_{1}, 2_{1}, 3_{1}\right) \\
\left(1_{2} ; 4_{1}, 0_{1}, 1_{1}, 2_{1}, 3_{1}\right),\left(1_{2} ; 2_{2}, 4_{1}, 5_{1}, 6_{1}, 7_{1}\right) \\
\text { and }\left(2_{2} ; 5_{1}, 0_{1}, 1_{1}, 2_{1}, 3_{1}\right)\end{array}$ & $\begin{array}{c}\left(0_{2} ; 1_{2}, 4_{1}, 5_{1}, 6_{1}, 7_{1}\right),\left(0_{2} ; 1_{2}, 0_{1}, 1_{1}, 2_{1}, 3_{1}\right) \\
\left(1_{2} ; 0_{2}, 0_{1}, 1_{1}, 2_{1}, 3_{1}\right),\left(1_{2} ; 0_{2}, 4_{1}, 5_{1}, 6_{1}, 7_{1}\right) \\
\text { and }\left(2_{2} ; 1_{2}, 0_{1}, 1_{1}, 2_{1}, 3_{1}\right)\end{array}$ & $E_{5}$ \\
\hline $\begin{array}{c}\left(0_{2} ; 0_{1}, 4_{1}, 5_{1}, 6_{1}, 7_{1}\right),\left(0_{2} ; 4_{1}, 0_{1}, 1_{1}, 2_{1}, 3_{1}\right), \\
\left(1_{2} ; 4_{1}, 0_{1}, 1_{1}, 2_{1}, 3_{1}\right), \text { and }\left(1_{2} ; 8_{1}, 4_{1}, 5_{1}, 6_{1}, 7_{1}\right)\end{array}$ & $\begin{array}{c}\left(0_{2} ; 1_{2}, 4_{1}, 5_{1}, 6_{1}, 7_{1}\right),\left(0_{2} ; 1_{2}, 0_{1}, 1_{1}, 2_{1}, 3_{1}\right) \\
\left(1_{2} ; 0_{2}, 0_{1}, 1_{1}, 2_{1}, 3_{1}\right), \text { and }\left(1_{2} ; 0_{2}, 4_{1}, 5_{1}, 6_{1}, 7_{1}\right)\end{array}$ & $E_{6}$ \\
\hline $\begin{array}{c}\left(0_{2} ; 2_{2}, 4_{1}, 5_{1}, 6_{1}, 7_{1}\right),\left(1_{2} ; 2_{2}, 0_{1}, 1_{1}, 2_{1}, 3_{1}\right) \\
\left(1_{2} ; 3_{2}, 4_{1}, 5_{1}, 6_{1}, 7_{1}\right), \text { and }\left(3_{2} ; 4_{2}, 4_{1}, 5_{1}, 6_{1}, 7_{1}\right)\end{array}$ & $\begin{array}{c}\left(0_{2} ; 1_{2}, 4_{1}, 5_{1}, 6_{1}, 7_{1}\right),\left(1_{2} ; 0_{2}, 0_{1}, 1_{1}, 2_{1}, 3_{1}\right) \\
\left(1_{2} ; 0_{2}, 4_{1}, 5_{1}, 6_{1}, 7_{1}\right), \text { and }\left(3_{2} ; 1_{2}, 4_{1}, 5_{1}, 6_{1}, 7_{1}\right)\end{array}$ & $E_{7}$ \\
\hline $\begin{array}{c}\left(0_{2} ; 2_{2}, 4_{1}, 5_{1}, 6_{1}, 7_{1}\right),\left(1_{2} ; 2_{2}, 0_{1}, 1_{1}, 2_{1}, 3_{1}\right) \\
\text { and }\left(1_{2} ; 3_{2}, 4_{1}, 5_{1}, 6_{1}, 7_{1}\right)\end{array}$ & $\begin{array}{c}\left(0_{2} ; 1_{2}, 4_{1}, 5_{1}, 6_{1}, 7_{1}\right),\left(1_{2} ; 0_{2}, 0_{1}, 1_{1}, 2_{1}, 3_{1}\right) \\
\text { and }\left(1_{2} ; 0_{2}, 4_{1}, 5_{1}, 6_{1}, 7_{1}\right)\end{array}$ & $E_{8}$ \\
\hline $\begin{array}{c}\left(0_{2} ; 0_{1}, 4_{1}, 5_{1}, 6_{1}, 7_{1}\right),\left(0_{2} ; 4_{1}, 0_{1}, 1_{1}, 2_{1}, 3_{1}\right), \\
\left(1_{2} ; 5_{1}, 0_{1}, 1_{1}, 2_{1}, 3_{1}\right), \text { and }\left(1_{2} ; 1_{1}, 4_{1}, 5_{1}, 6_{1}, 7_{1}\right)\end{array}$ & $\begin{array}{c}\left(0_{2} ; 1_{2}, 4_{1}, 5_{1}, 6_{1}, 7_{1}\right),\left(0_{2} ; 1_{2}, 0_{1}, 1_{1}, 2_{1}, 3_{1}\right) \\
\left(1_{2} ; 0_{2}, 0_{1}, 1_{1}, 2_{1}, 3_{1}\right), \text { and }\left(1_{2} ; 0_{2}, 4_{1}, 5_{1}, 6_{1}, 7_{1}\right)\end{array}$ & $E_{9}$ \\
\hline $\begin{array}{c}\left(0_{2} ; 0_{1}, 4_{1}, 5_{1}, 6_{1}, 7_{1}\right),\left(0_{2} ; 4_{1}, 0_{1}, 1_{1}, 2_{1}, 3_{1}\right) \\
\left(1_{2} ; 5_{1}, 0_{1}, 1_{1}, 2_{1}, 3_{1}\right),\left(1_{2} ; 2_{2}, 4_{1}, 5_{1}, 6_{1}, 7_{1}\right), \\
\quad \text { and }\left(2_{2} ; 6_{1}, 0_{1}, 1_{1}, 2_{1}, 3_{1}\right)\end{array}$ & $\begin{array}{c}\left(0_{2} ; 1_{2}, 4_{1}, 5_{1}, 6_{1}, 7_{1}\right),\left(0_{2} ; 1_{2}, 0_{1}, 1_{1}, 2_{1}, 3_{1}\right) \\
\left(1_{2} ; 0_{2}, 0_{1}, 1_{1}, 2_{1}, 3_{1}\right),\left(1_{2} ; 0_{2}, 4_{1}, 5_{1}, 6_{1}, 7_{1}\right) \\
\text { and }\left(2_{2} ; 1_{2}, 0_{1}, 1_{1}, 2_{1}, 3_{1}\right)\end{array}$ & $E_{10}$ \\
\hline$\left(0_{2} ; 0_{1}, 4_{1}, 5_{1}, 6_{1}, 7_{1}\right)$ & $\left(0_{2} ; 1_{2}, 4_{1}, 5_{1}, 6_{1}, 7_{1}\right)$ & $E_{13}$ \\
\hline$\left(1_{2} ; 2_{2}, 4_{1}, 5_{1}, 6_{1}, 7_{1}\right)$ and $\left(2_{2} ; 4_{1}, 0_{1}, 1_{1}, 2_{1}, 3_{1}\right)$ & $\left(1_{2} ; 0_{2}, 4_{1}, 5_{1}, 6_{1}, 7_{1}\right)$ and $\left(2_{2} ; 1_{2}, 0_{1}, 1_{1}, 2_{1}, 3_{1}\right)$ & $E_{14}$ \\
\hline$\left(0_{2} ; 0_{1}, 4_{1}, 5_{1}, 6_{1}, 7_{1}\right)$ and $\left(0_{2} ; 0_{1}, 8_{1}, 2_{2}, 3_{2}, 4_{2}\right)$ & $\left(0_{2} ; 1_{2}, 4_{1}, 5_{1}, 6_{1}, 7_{1}\right)$ and $\left(0_{2} ; 1_{2}, 8_{1}, 2_{2}, 3_{2}, 4_{2}\right)$ & $E_{15}$ \\
\hline $\begin{array}{c}\left(1_{2} ; 2_{2}, 4_{1}, 5_{1}, 6_{1}, 7_{1}\right),\left(2_{2} ; 3_{2}, 0_{1}, 1_{1}, 2_{1}, 3_{1}\right) \\
\left(1_{2} ; 3_{2}, 0_{1}, 1_{1}, 2_{1}, 3_{1}\right), \text { and }\left(3_{2} ; 2_{2}, 4_{1}, 5_{1}, 6_{1}, 7_{1}\right)\end{array}$ & $\begin{array}{c}\left(1_{2} ; 0_{2}, 4_{1}, 5_{1}, 6_{1}, 7_{1}\right),\left(2_{2} ; 1_{2}, 0_{1}, 1_{1}, 2_{1}, 3_{1}\right) \\
\left(1_{2} ; 0_{2}, 0_{1}, 1_{1}, 2_{1}, 3_{1}\right), \text { and }\left(3_{2} ; 1_{2}, 4_{1}, 5_{1}, 6_{1}, 7_{1}\right)\end{array}$ & $E_{16}$ \\
\hline $\begin{array}{c}\left(1_{2} ; 2_{2}, 4_{1}, 5_{1}, 6_{1}, 7_{1}\right),\left(1_{2} ; 3_{2}, 0_{1}, 1_{1}, 2_{1}, 3_{1}\right), \\
\text { and }\left(3_{2} ; 2_{2}, 4_{1}, 5_{1}, 6_{1}, 7_{1}\right)\end{array}$ & $\begin{array}{c}\left(1_{2} ; 0_{2}, 4_{1}, 5_{1}, 6_{1}, 7_{1}\right),\left(1_{2} ; 0_{2}, 0_{1}, 1_{1}, 2_{1}, 3_{1}\right), \\
\text { and }\left(3_{2} ; 1_{2}, 4_{1}, 5_{1}, 6_{1}, 7_{1}\right)\end{array}$ & $E_{17}$ \\
\hline $\begin{array}{c}\left(1_{2} ; 2_{2}, 4_{1}, 5_{1}, 6_{1}, 7_{1}\right),\left(1_{2} ; 4_{2}, 0_{1}, 1_{1}, 2_{1}, 3_{1}\right), \\
\text { and }\left(4_{2} ; 3_{2}, 0_{1}, 1_{1}, 2_{1}, 3_{1}\right)\end{array}$ & $\begin{array}{c}\left(1_{2} ; 0_{2}, 4_{1}, 5_{1}, 6_{1}, 7_{1}\right),\left(1_{2} ; 0_{2}, 0_{1}, 1_{1}, 2_{1}, 3_{1}\right), \\
\text { and }\left(4_{2} ; 1_{2}, 0_{1}, 1_{1}, 2_{1}, 3_{1}\right)\end{array}$ & $E_{18}$ \\
\hline $\begin{array}{c}\left(1_{2} ; 2_{2}, 4_{1}, 5_{1}, 6_{1}, 7_{1}\right),\left(2_{2} ; 4_{1}, 0_{1}, 1_{1}, 2_{1}, 3_{1}\right) \\
\left(1_{2} ; 4_{2}, 0_{1}, 1_{1}, 2_{1}, 3_{1}\right) \text { and }\left(4_{2} ; 3_{2}, 0_{1}, 1_{1}, 2_{1}, 3_{1}\right)\end{array}$ & $\begin{array}{c}\left(1_{2} ; 0_{2}, 4_{1}, 5_{1}, 6_{1}, 7_{1}\right),\left(2_{2} ; 1_{2}, 0_{1}, 1_{1}, 2_{1}, 3_{1}\right) \\
\left(1_{2} ; 0_{2}, 0_{1}, 1_{1}, 2_{1}, 3_{1}\right) \text { and }\left(4_{2} ; 1_{2}, 0_{1}, 1_{1}, 2_{1}, 3_{1}\right)\end{array}$ & $E_{19}$ \\
\hline $\begin{array}{c}\left(1_{2} ; 3_{2}, 4_{1}, 5_{1}, 6_{1}, 7_{1}\right),\left(3_{2} ; 2_{2}, 4_{1}, 5_{1}, 6_{1}, 7_{1}\right) \\
\left(1_{2} ; 4_{2}, 0_{1}, 1_{1}, 2_{1}, 3_{1}\right) \text { and }\left(4_{2} ; 3_{2}, 0_{1}, 1_{1}, 2_{1}, 3_{1}\right)\end{array}$ & $\begin{array}{c}\left(1_{2} ; 0_{2}, 4_{1}, 5_{1}, 6_{1}, 7_{1}\right),\left(3_{2} ; 1_{2}, 4_{1}, 5_{1}, 6_{1}, 7_{1}\right) \\
\left(1_{2} ; 0_{2}, 0_{1}, 1_{1}, 2_{1}, 3_{1}\right) \text { and }\left(4_{2} ; 1_{2}, 0_{1}, 1_{1}, 2_{1}, 3_{1}\right)\end{array}$ & $E_{20}$ \\
\hline$\left(0_{2} ; 0_{1}, 4_{1}, 5_{1}, 6_{1}, 7_{1}\right)$ and $\left(1_{2} ; 4_{1}, 0_{1}, 1_{1}, 2_{1}, 3_{1}\right)$ & $\left(0_{2} ; 1_{2}, 4_{1}, 5_{1}, 6_{1}, 7_{1}\right)$ and $\left(1_{2} ; 0_{2}, 0_{1}, 1_{1}, 2_{1}, 3_{1}\right)$ & $E_{21}$ \\
\hline$\left(0_{2} ; 0_{1}, 4_{1}, 5_{1}, 6_{1}, 7_{1}\right)$ and $\left(0_{2} ; 1_{1}, 8_{1}, 2_{2}, 3_{2}, 4_{2}\right)$ & $\left(0_{2} ; 1_{2}, 4_{1}, 5_{1}, 6_{1}, 7_{1}\right)$ and $\left(0_{2} ; 1_{2}, 8_{1}, 2_{2}, 3_{2}, 4_{2}\right)$ & $E_{22}$ \\
\hline$\left(0_{2} ; 0_{1}, 4_{1}, 5_{1}, 6_{1}, 7_{1}\right)$ and $\left(1_{2} ; 0_{1}, 4_{1}, 5_{1}, 6_{1}, 7_{1}\right)$ & $\left(0_{2} ; 1_{2}, 4_{1}, 5_{1}, 6_{1}, 7_{1}\right)$ and $\left(1_{2} ; 0_{2}, 4_{1}, 5_{1}, 6_{1}, 7_{1}\right)$ & $E_{23}$ \\
\hline
\end{tabular}

Table 3: Substitutions to obtain different excesses for $n=14$ 
In order to achieve the excess $E_{11}$, write $K_{14}=K_{4} \vee K_{10}$. Label the vertices of $K_{10}$ by the elements of $\mathbb{Z}_{10}$ having subscript 1 and the vertices of $K_{4}$ with the elements of $\mathbb{Z}_{4}$ having subscript 2. The complete graph $K_{10}$ has an $S_{5}$-decomposition, $U$. The vertices $0_{1}, 1_{1}, 2_{1}, 3_{1}$, and $4_{1}$, the vertices $0_{2}, 1_{2}, 2_{2}$, and $3_{2}$, and the edges between these sets form a complete bipartite graph with one part of size a multiple of 5 . Hence, by Lemma 3, this bipartite graph has an $S_{5}$-decomposition, $V$. Moreover, the following stars form a minimum covering, $W$, for the remaining graph with the edges $\left\{8_{1}, 0_{1}\right\},\left\{9_{1}, 1_{1}\right\},\left\{0_{2}, 2_{2}\right\}$, and $\left\{1_{2}, 3_{2}\right\}$ as the excess.

$$
\begin{aligned}
& \left(0_{2} ; 1_{2}, 2_{2}, 5_{1}, 6_{1}, 7_{1}\right),\left(1_{2} ; 2_{2}, 3_{2}, 5_{1}, 6_{1}, 7_{1}\right),\left(2_{2} ; 3_{2}, 0_{2}, 5_{1}, 6_{1}, 7_{1}\right), \\
& \left(3_{2} ; 0_{2}, 1_{2}, 5_{1}, 6_{1}, 7_{1}\right),\left(8_{1} ; 0_{1}, 0_{2}, 1_{2}, 2_{2}, 3_{2}\right),\left(9_{1} ; 1_{1}, 0_{2}, 1_{2}, 2_{2}, 3_{2}\right) .
\end{aligned}
$$

Therefore, $U \cup V \cup W$ is a minimum covering of $K_{14}$ with the excess $E_{11}$ (see Figure 8). In

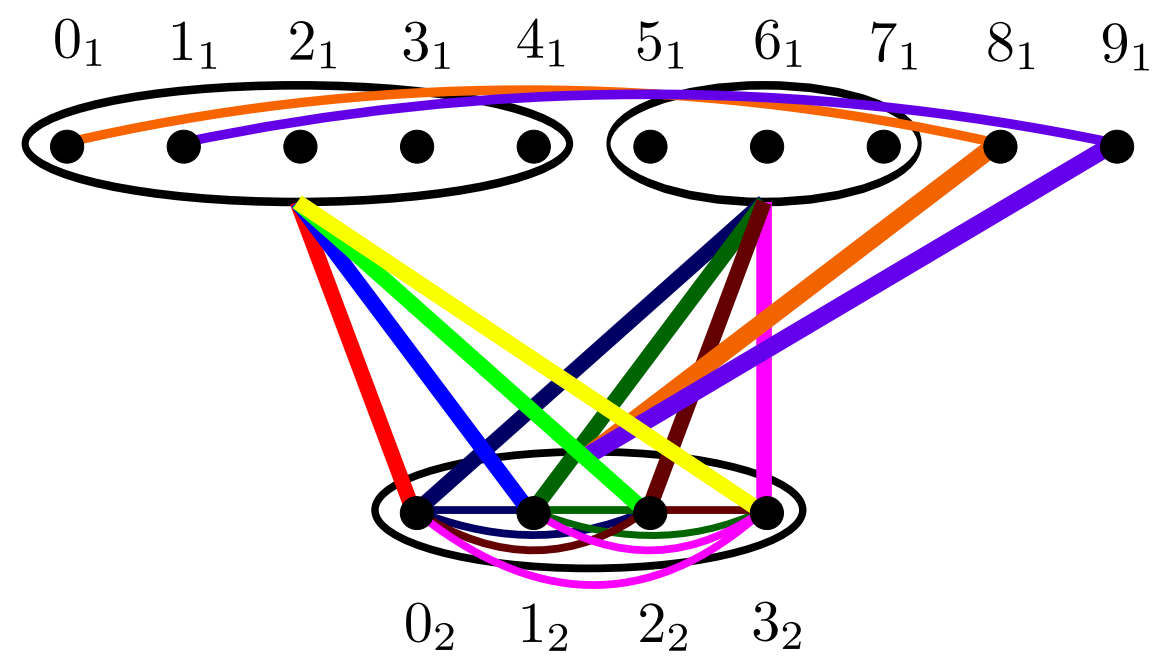

Figure 8: Minimum covering of $K_{14}$ with the excess $E_{11}$

order to achieve the excess $E_{12}$ for $n \geq 17$ where $n \equiv 2(\bmod 5)$, it suffices to achieve this excess for $n=17$ by Lemma 8. Let $n=17$. Write $K_{17}=K_{3} \vee K_{14}$, label the vertices of $K_{14}$ with the elements of $\mathbb{Z}_{14}$ having subscript 1 and the vertices of $K_{3}$ with the elements of $\mathbb{Z}_{3}$ having subscript 2 . As shown in case 3 , the graph $K_{14}$ has an $S_{5}$-covering, $R$, with the excess $E_{12}$. The vertices $0_{1}, 1_{1}, \ldots, 9_{1}$, the vertices $0_{2}, 1_{2}, 2_{2}$, and the edges between these two sets form a complete bipartite graph with one part of size a multiple of 5 . Hence, by Lemma 3, this complete bipartite graph has an $S_{5}$-decomposition, $S$. Now, the vertices $10_{1}, 11_{1}, 12_{1}, 13_{1}$, the vertices $0_{2}, 1_{2}, 2_{2}$, the edges between these two sets, and the edges within the second set form a graph $K_{3} \vee K_{4}$, which has an $S_{5}$-decomposition, $T$, by Lemma 4 . Therefore, $R \cup S \cup T$ forms a minimum $S_{5}$-covering of $K_{17}$ with the excess $E_{12}$. 


\begin{tabular}{ccc}
\hline \hline$n(\bmod 10)$ & Possible Leaves & Possible Excesses \\
\hline 0 & $\emptyset$ & $\emptyset$ \\
\hline 1 & $\emptyset$ & $\emptyset$ \\
\hline 2 & $K_{2}$ & the graphs in Figure 3 \\
\hline 3 & $S_{3}, K_{3}, P_{4}, 3 K_{2}$, and $S_{2}+K_{2}$ & $S_{2}, 2 K_{2}$, and $K_{2}^{2}$ \\
\hline 4 & $K_{2}$ & the graphs in Figure 3 \\
\hline 5 & $\emptyset$ & $\emptyset$ \\
\hline 6 & $\emptyset$ & the graphs in Figure 3 \\
\hline 7 & $K_{2}$ & $S_{2}, 2 K_{2}$, and $K_{2}^{2}$ \\
\hline 8 & $S_{3}, K_{3}, P_{4}, 3 K_{2}$, and $S_{2}+K_{2}$ & the graphs in Figure 3 \\
\hline 9 & $K_{2}$ &
\end{tabular}

Table 4: All possible leaves (excesses) for $S_{5}$-packings $\left(S_{5}\right.$-coverings) of the complete graph

\section{Conclusions and Future Directions}

In this paper, we have solved the spectrum problem for packings and coverings of the complete graph with stars with up to five edges. It is tempting to investigate for a generalization of the existing results. As the very next step, we are going to consider the spectrum problem for packings and coverings of complete graphs on some specific number of vertices with $k$-stars for any positive integer $k$.

Additionally, we are planning to work on the spectrum problem for packings and coverings of the complete graph with all trees with up to five edges. As another direction, we are interested in investigating different possibilities for leaves and excesses of maximal packings (minimal coverings) of the complete graph with trees with up to five edges.

\section{References}

[1] Adams P., Bryant D., Buchanan M.: A survey on the existence of $G$-designs. J. Combin. Des. 16, 373-410 (2008).

[2] Adams P., Bryant D., El-Zanati S.: Packing and covering the complete graph with cubes. Australas. J. Combin. 20, 267288 (1999).

[3] Bryant D.: Packing paths in complete graphs. J. Combin. Theory Ser. B 100 (no. 2), 206215 (2010).

[4] Bryant D., Horsley D.: Packing cycles in complete graphs. J. Combin. Theory Ser. B 98 (no. 5), 10141037 (2008).

[5] Colbourn C., Dinitz J.:The CRC Handbook of Combinatorial Designs (second edition), Chapter 24, CRC Press, Boca Raton (2007).

[6] Dyer D., Haghshenas S., Shalaby N.: Constructing the spectrum of packings and coverings for the complete graph with 4-stars. Submitted to Discrete Math. 
[7] Hell P., Rosa A.: Graph decompositions, handcuffed prisoners and balanced P-designs. Discrete Math. 2 (no. 3), 229-252 (1972).

[8] Hoffman, D.G., Roberts, D.: Maximum packings of $K_{n}$ with $k$-stars. Australas. J. Combin. 59, 206-210 (2014).

[9] Huang C., Rosa A.: Decomposition of complete graphs into trees. Ars Combinatoria 5, 23-63 (1978).

[10] Roditty Y.: Packing and covering of the complete graph with a graph $G$ of four vertices or less. J. Combin. Theory Ser. B 34 (no. 2), 231-243 (1983).

[11] Roditty Y.: Packing and covering of the complete graph. II. The trees of order six. Ars Combin. 19, 81-93 (1985).

[12] Roditty Y.: The packing and covering of the complete graph. I. The forests of order five. Int. J. Math. Math. Sci. 9 (no. 2), 277-282 (1986).

[13] Roditty Y.: Packing and covering of the complete graph. IV. The trees of order seven. Ars Combin. 35, 33-64 (1993).

[14] Tarsi M.: Decomposition of complete multigraphs into stars. Discrete Math. 26, 273-278 (1979).

[15] Tarsi M.: Decomposition of a complete multigraph into simple paths: nonbalanced handcuffed designs. J. Combin. Theory Ser. A 34 (no. 1), 6070 (1983).

[16] West D.: Introduction to Graph Theory (second edition). Prentice Hall (2001).

[17] Wilson R.: Decompositions of complete graphs into subgraphs isomorphic to a given graph, Congr. Numer. 15, 647-659 (1976).

[18] Yamamoto S., Ikedo H., Shige-eda S., Ushio K., Hamada N.: On claw-decompositions of complete graphs and complete bigraphs. Hiroshima Math. J. 5, 33-42 (1975). 